\title{
Quantum Monte-Carlo method without negative-sign problem for two-dimensional electron systems under strong magnetic fields
}

\author{
Sei Suzuki* and Tatsuya Nakajima ${ }^{1}$ \\ Department of Basic Science, University of Tokyo, Tokyo 153-8902 \\ ${ }^{1}$ Department of Physics, Tohoku University, Sendai 980-8578
}

(Received November 19, 2018)

\begin{abstract}
The quantum Monte-Carlo method is applied to two-dimensional electron systems under strong magnetic fields. The negative-sign problem involved by this method can be avoided for certain filling factors by modifying interaction parameters from those of the Coulomb interaction. Our techniques for obtaining sign-problem-free parameters are described in detail. Calculated results on static observables are also reported for Landau level filling $\nu=1 / 3$.
\end{abstract}

KEYWORDS: quantum Monte-Carlo, negative-sign problem, linear programming, fractional quantum Hall states

It has been revealed that a two-dimensional electron system in a strong magnetic field has various groundstate phases. In particular, the existence of incompressible liquids gives rise to the fractional quantum Hall effect ${ }^{1-3}$ and has attracted much theoretical and experimental interests to this so-called quantum Hall $(\mathrm{QH})$ system. Rich quantum phases realized in the $\mathrm{QH}$ system are attributed to Coulomb interactions between electrons, because the kinetic energy is quenched by the Landau quantization in a strong magnetic field. Thus the ground state involves strong electronic correlations and is difficult to consider by use of mean field theory.

So far several many-electron states have been proposed theoretically for the ground state in each phase. The Laughlin state, ${ }^{4}$ Jain state, ${ }^{5}$ and Pfaffian state ${ }^{6}$ successfully account for incompressible liquid phases in the $\mathrm{QH}$ system. On the other hand, the composite-fermion liquid states $^{7,8}$ and anisotropic charge-density-wave states ${ }^{9}$ are plausible to compressible phases at the half filling of the lowest and higher Landau levels, respectively.

When the properties of such strongly-interacting systems are investigated, numerical studies can provide a variety of significant information. The exact diagonalization method of the Hamiltonian matrix has been frequently used to inspect the various properties of the $\mathrm{QH}$ system $^{10}$ so far, but its applicability is limited to smallsize systems. Recently the density matrix renormalization group (DMRG) method has been applied to the study of the ground-state properties of the QH system. ${ }^{11}$ The system size permitted in the DMRG method is much larger than that in the exact diagonalization study, but it is difficult to study dynamical quantities by the DMRG method.

In the present study, we propose an application of the quantum Monte-Carlo (QMC) method to the QH system. This is because the QMC method can investigate not only static but also dynamical quantities in largesize systems. This method can be applied for both zeroand finite-temperature cases and has ever been used for a variety of quantum many-body systems. However, the
QMC method is accompanied by the negative-sign problem, which sometimes makes us away from investigating physical properties. Thus we first need to resolve the negative-sign problem in order to make the best use of the present method. In this letter, we present one solution to the negative-sign problem in the $\mathrm{QH}$ system.

This letter is organized as follows. We first express physical quantities in the $\mathrm{QH}$ system in terms of the auxiliary-field path-integral. Then we describe how to avoid the negative-sign problem in the present system. Finally some results of QMC calculations are presented.

The system studied here is composed of interacting electrons confined on a spherical surface. ${ }^{12}$ It is assumed that a magnetic monopole is located at the center of the sphere. The monopole induces a uniform magnetic field on the surface. The number of flux quanta diverging from the monopole is denoted by $2 s$ hereafter $(2 s$ : integer). Single-electron states on the sphere are specified by the Landau level index and the $z$-component quantum number, $m$, of angular momentum (AM). We consider the strong magnetic-field limit and restrict the single-electron states to the subspace of the lowest Landau level (LLL). Then a single-electron state is specified by only $m$ which ranges from $-s$ to $s$, and the number of degenerate single-electron states amounts to $N_{s}=2 s+1$. The spin degrees of freedom are neglected for simplicity.

In the LLL approximation, the kinetic energy is constant for $\mathrm{QH}$ systems containing the fixed number of electrons. The remaining term of the Hamiltonian comes from Coulomb interactions between electrons. We express the interaction Hamiltonian in a bilinear form of the density operator to introduce the HubbardStratonovich transformation needed for QMC calculations.

The density operator is defined using the Clebsch-Gordan coefficient as $\rho_{K N}=$ $\sum_{m_{1}=-s}^{s} \sum_{m_{2}=-s}^{s}\left\langle K N \mid s m_{1} ; s m_{2}\right\rangle a_{m_{1}}^{\dagger} \tilde{a}_{m_{2}}$, where $a_{m}$ is the annihilation operator of an electron with AM quantum number $m$ and $\tilde{a}_{m}=(-1)^{s+m} a_{-m}$ is the time reversal of $a_{m}$. The interaction Hamiltonian is written 
in terms of the density operator as

$\mathcal{H}=-\frac{1}{2} \sum_{K=0}^{2 s} \chi_{K} \sum_{N=-K}^{K}(-1)^{K+N} \rho_{K N} \rho_{K-N}+\epsilon_{0} \rho_{00}$.

Here $\epsilon_{0}=-\sum_{J=0}^{2 s}(-1)^{2 s-J}(2 J+1) V_{J} /(2 \sqrt{2 s+1})$ and the coefficient $\chi_{K}$ is given in terms of the Haldane pseudopotential, ${ }^{13} V_{J}$, as $\chi_{K}=\sum_{J=0}^{2 s} T_{K J} V_{J}$. The matrix $T$ is explicitly written using Wigner's $6 j$ symbol as $T_{K J}=\sum_{J=0}^{2 s}(-1)^{2 s-J+K+1}(2 J+1)\left\{\begin{array}{c}s s J \\ s s K\end{array}\right\}$.

The interaction Hamiltonian can also be written in another form as $\mathcal{H}=\frac{1}{2} \sum_{J=0}^{2 s} V_{J} \sum_{M=-J}^{J} A_{J M}^{\dagger} A_{J M}$, where $A_{J M}$ is the pairing operator given by $A_{J M}=$ $\sum_{m_{1}=-s}^{s} \sum_{m_{2}=-s}^{s}\left\langle J M \mid s m_{1} ; s m_{2}\right\rangle a_{m_{1}} a_{m_{2}}$. Since $a_{m}$ satisfies the anti-commutation relation, $A_{J M}=0$ for even $2 s-J$. Thus the terms with $V_{J}$ for even $2 s-J$ are unphysical and have no influence on physical quantities. Therefore these unphysical pseudopotentials can be used as tunable parameters to improve the efficiency of QMC calculations.

The imaginary-time evolution operator $e^{-\beta \mathcal{H}}$ is decomposed to the product of imaginary-time slices $e^{-\Delta \beta \mathcal{H}}(\beta$ : inverse temperature, $\Delta \beta \equiv \beta / N_{\mathrm{t}}, N_{\mathrm{t}}$ : Trotter number). By introducing the Hubbard-Stratonovich transformation for each slice, the evolution operator $e^{-\beta \mathcal{H}}$ can be written in terms of a linearized Hamiltonian given by

$$
\begin{aligned}
h(\sigma)= & \frac{1}{2} \sum_{K=1}^{2 s} \eta_{K} \chi_{K}\left[\sum_{N=1}^{K} 2\left(\sigma_{K N}^{*} \rho_{K N}+\sigma_{K N} \tilde{\rho}_{K N}\right)\right. \\
& \left.+\sigma_{K 0}^{*} \rho_{K 0}+\sigma_{K 0} \tilde{\rho}_{K 0}\right]+\frac{1}{2} \chi_{0}\left(\rho_{00}\right)^{2}+\epsilon_{0} \rho_{00} \cdot
\end{aligned}
$$

Here $\tilde{\rho}_{K N}=(-1)^{K} \rho_{K N}^{\dagger}, \sigma_{K N}$ is an auxiliary field for a mode $(K, N)$ introduced by the Hubbard-Stratonovich transformation, and $\eta_{K}$ is a numerical factor which takes 1 for non-negative $\chi_{K}$ and $i$ for negative one. We remark that the terms, $\chi_{00}\left(\rho_{00}\right)^{2} / 2$ and $\epsilon_{0} \rho_{00}$, in Eq. (2) are constants because $\rho_{00}$ is proportional to the fixed number of electrons.

The partition functions, $Z=\operatorname{Tr}\left[e^{-\beta \mathcal{H}}\right]$ for canonical ensemble and $Z=\left\langle\psi\left|e^{-\beta \mathcal{H}}\right| \psi\right\rangle$ for zero temperature, are represented in terms of auxiliary-field pathintegral as $Z=\int \mathcal{D} \sigma \zeta(\sigma)$. Here the weight function is defined by $\zeta(\sigma) \equiv \operatorname{Tr}[U(\beta ; 0)]$ for canonical ensemble and by $\zeta(\sigma) \equiv\langle\psi|U(\beta ; 0)| \psi\rangle$ for zero temperature, $U(\beta ; 0)$ in the weight function is defined by $U(\beta ; 0) \equiv e^{-\Delta \beta h\left(\sigma^{\left(N_{t}\right)}\right)} \cdots e^{-\Delta \beta h\left(\sigma^{(1)}\right)}$, and $|\psi\rangle$ in the zero-temperature formalism is an arbitrary state not orthogonal to the true ground state. The expectation value of an observable $\mathcal{O}$ is written in the path-integral representation as $\langle\mathcal{O}\rangle=\int \mathcal{D} \sigma\langle\mathcal{O}\rangle_{\sigma} \zeta(\sigma) / Z$, where $\langle\mathcal{O}\rangle_{\sigma} \equiv$ $\operatorname{Tr}[\mathcal{O} U(\beta ; 0)] / \zeta(\sigma)$ for canonical ensemble and $\langle\mathcal{O}\rangle_{\sigma}=$ $\langle\psi|U(\beta ; \beta / 2) \mathcal{O} U(\beta / 2 ; 0)| \psi\rangle / \zeta(\sigma)$ for zero temperature.

The auxiliary-field path-integral is evaluated by means of the Metropolis-Monte-Carlo technique. However, the weight function, $\zeta(\sigma)$, can be negative for certain configurations of auxiliary fields. Thus let us regard $P(\sigma) \equiv|\zeta(\sigma)|$ as a positive-definite probability distribution. The expectation value is then written as $\langle\mathcal{O}\rangle=$ $\int \mathcal{D} \sigma\left[\langle\mathcal{O}\rangle_{\sigma} \xi(\sigma)\right] P(\sigma) / \int \mathcal{D} \sigma \xi(\sigma) P(\sigma)$, where $\xi(\sigma) \equiv$ $\zeta(\sigma) /|\zeta(\sigma)|$ is the sign of $\zeta(\sigma)$. If the expectation value of $\xi(\sigma)$ is vanishingly small, Monte-Carlo evaluations of $\langle\mathcal{O}\rangle$ become very unstable. This is the so-called negative-sign problem. Thus, in order to make a precise evaluation, it is desirable that $\zeta(\sigma)$ is always positive.

For detailed discussion on the negative-sign problem, let us move to the matrix representation of the QMC method. ${ }^{14}$ In order to exploit the timereversal symmetry, we first define fermionic operators, $\alpha_{m}$, by $\alpha_{m}=a_{m}$ for $m \geq 0$, and $\alpha_{m}=$ $(-1)^{s-m} a_{m}$ for $m<0$. By introducing a matrix $\mathbf{M}(\sigma)$ as $-\Delta \beta h(\sigma)=\sum_{m, n=-s}^{s} M_{m n}(\sigma) \alpha_{m}^{\dagger} \alpha_{n}$, we define $\mathbf{U}(\beta ; 0) \equiv e^{\mathbf{M}\left(\sigma^{\left(N_{t}\right)}\right)} \cdots e^{\mathbf{M}\left(\sigma^{(1)}\right)}$. The weight function in the canonical ensemble is produced by operating the particle-number projection to that in the groundcanonical ensemble: ${ }^{15}$

$\zeta(\sigma)=\sum_{I=1}^{N_{s}} e^{-2 \pi i N_{e}\left(I / N_{s}\right)} \operatorname{det}\left[\mathbf{1}+e^{2 \pi i\left(I / N_{s}\right)} \mathbf{U}(\beta ; 0)\right]$,

where $N_{e}$ is the number of electrons. In the zerotemperature formalism, the weight function is given by $\zeta(\sigma)=\operatorname{det}\left[\mathbf{V}^{\dagger} \mathbf{U}(\beta ; 0) \mathbf{V}\right]$, where an arbitrary state is written in terms of a matrix $\mathbf{V}$ and electron vacuum $|0\rangle$ as $|\psi\rangle=\left(\sum_{m=-s}^{s} \alpha_{m}^{\dagger} V_{m 1}\right) \cdots\left(\sum_{m=-s}^{s} \alpha_{m}^{\dagger} V_{m N_{e}}\right)|0\rangle$. We remark that $\mathbf{M}$ and $\mathbf{U}$ are square matrices of dimension $N_{s}$, while $\mathbf{V}$ is a $N_{s} \times N_{e}$ rectangular matrix.

For the zero-temperature formalism, the negative-sign problem is overcome under the following conditions: ${ }^{16}$

(i) $2 s$ is odd and $N_{e}$ is even.

(ii) $\chi_{K}$ is non-negative for $K=1,2, \cdots, 2 s$.

By the condition (i), the dimension, $N_{s}$, of matrix $\mathbf{M}$ becomes even. The condition (ii) yields $\eta_{K}=1$ in Eq.(2) and then $\mathbf{M}$ satisfies $M_{-m_{1},-m_{2}}=M_{m_{1}, m_{2}}^{*}$ and $M_{-m_{1}, m_{2}}=-M_{m_{1},-m_{2}}^{*}$ for positive $m_{1}$ and $m_{2}$. Therefore, by arranging AM indices, $m$, in the matrix representation as $m=s, \cdots, 1 / 2,-s, \cdots,-1 / 2, \mathbf{M}$ has such a form as

$$
\mathbf{M}=\left[\begin{array}{cc}
\mathbf{A} & \mathbf{B} \\
-\mathbf{B}^{*} & \mathbf{A}^{*}
\end{array}\right]
$$

where $\mathbf{A}$ and $\mathbf{B}$ are square block matrices of dimension $N_{s} / 2$. When matrices $\mathbf{M}_{1}$ and $\mathbf{M}_{2}$ are of the form of Eq.(4), the product $\mathbf{M}_{1} \mathbf{M}_{2}$ is also of the form of Eq.(4). Thus $\mathbf{U}(\beta ; 0)$ also has the form of Eq.(4). When we choose a matrix $\mathbf{V}$ that satisfies $V_{-m, N_{e} / 2+n}=V_{m, n}^{*}$ and $V_{-m, n}=-V_{m, N_{e} / 2+n}^{*}$ for positive $m$ and $1 \leq n \leq N_{e} / 2$, the following property is also obtained:

$$
\mathbf{V}^{\dagger} \mathbf{U}(\beta ; 0) \mathbf{V}=\left[\begin{array}{cc}
\mathbf{P} & \mathbf{Q} \\
-\mathbf{Q}^{*} & \mathbf{P}^{*}
\end{array}\right]
$$

where $\mathbf{P}$ and $\mathbf{Q}$ are square block matrices of dimension $N_{e} / 2$. Then, if $\lambda_{i}$ is an eigenvalue of $\mathbf{V}^{\dagger} \mathbf{U}(\beta ; 0) \mathbf{V}$, its complex-conjugate is also shown to be its eigenvalue. Thus we obtain $\zeta(\sigma)=\prod_{i=1}^{N_{e} / 2} \lambda_{i} \lambda_{i}^{*} \geq 0$.

We can avoid the negative-sign disaster also in case of the canonical ensemble by realizing the following conditions: (i') $2 s$ is odd, and (ii) $\chi_{K} \geq 0$ for $K=1, \cdots, 2 s$. We note that even $N_{e}$ is not required in $\left(\mathrm{i}^{\prime}\right)$. Since the matrix $\mathbf{M}$ and $\mathbf{U}(\beta ; 0)$ then have the form of Eq. (4) as in the zero-temperature formalism, the weight function in the 
ground-canonical ensemble, $\operatorname{det}[\mathbf{1}+\mathbf{U}(\beta ; 0)]$, always becomes non-negative. The weight function in the canonical ensemble, which is obtained by operating the particlenumber projection to that of the ground-canonical ensemble [see Eq.(3)], is expected to be almost always positive. We have numerically confirmed that this is the case.

The next task is to inquire which systems satisfy the negative-sign-free conditions. The condition (i) $\left[\left(i^{\prime}\right)\right.$ for the canonical ensemble] restricts the Landau-level filling accessible by the QMC method. For instance, the $\nu=1 / m$ Laughlin state $(m$ : odd), characterized by $2 s=m\left(N_{e}-1\right)$, can be studied by the QMC method if $N_{e}$ is even. The condition (ii) brings about strong constraints for electron-electron interactions accessible by the QMC method. In fact, this condition is not satisfied by the Coulomb interaction within the LLL.

As mentioned above, the Haldane pseudopotentials, $V_{J}$, are classified into the physical components $(2 s-$ $J$ : odd) or unphysical ones $(2 s-J$ : even). We obtain coupling constants $\chi_{K}$ favorable to QMC calculations by using the unphysical ones as tunable parameters, with minimizing the difference of the physical ones between our model interaction and the Coulomb interaction. Then the model pseudopotentials are given in terms of obtained $\chi_{K}$ by $V_{J}=$ $\sum_{K=0}^{2 s} T_{J K} \chi_{K}$, because $\sum_{K} T_{J K} T_{K J^{\prime}}=\delta_{J J^{\prime}}$. We define the quantity to be minimized by $f\left(-\chi_{0}, \chi_{1}, \cdots, \chi_{2 s}\right)=$ $\sum_{2 s-J \text { :odd }} \kappa_{J}\left(\sum_{K=0}^{2 s} T_{J K} \chi_{K}-V_{J}^{\mathrm{C}}\right)$, where $V_{J}^{\mathrm{C}}$ is the pseudopotential of the Coulomb interaction and $\kappa_{J}$ controls the priority of each pseudopotential component. We note that the arguments of $f$ should be non-negative, which agrees with the condition (ii). Although the first argument, $-\chi_{0}$, is not referred by the condition (ii), it must be positive in order to make a repulsive pseudopotential $V_{0}=\sum_{K=0}^{2 s} T_{0 K} \chi_{K}>0$ under the conditions that $\chi_{K}>0$ for $1 \leq K \leq 2 s$ and $T_{0 K}<0$ for all $K$. Such a set of $\chi_{K}$ can be obtained by the linear programming method, which minimizes $f$ over its non-negative arguments imposing the inequalities $V_{J} \geq V_{J}^{\mathrm{C}}\left(V_{J} \leq V_{J}^{\mathrm{C}}\right)$ for non-negative (non-positive) $\kappa_{J}$.

A simple condition for the linear programming method is given by $\kappa_{J}=1$ for all of odd $2 s-J$. Then physical pseudopotentials are determined with equal weights and become larger than those of the Coulomb interaction, respectively. The coupling constants $\chi_{K}$ obtained in this way vanish except for the cases of $K=0$ and $K=1$. The Hamiltonian for this interaction is written as $H=-\frac{1}{2} \chi_{0} N_{e}^{2} / N_{s}+6 \chi_{1} \mathcal{L}^{2} /\left[\left(N_{s}-1\right) N_{s}\left(N_{s}+1\right)\right]$, where $\mathcal{L}$ denotes the total AM operator. The eigen-energies of this Hamiltonian depends only on the total AM quantum number. Therefore the ground state is energetically degenerate and the excitation spectrum is gapless.

Figure 1 shows the pseudopotentials of the Coulomb interaction and two negative-sign-free interactions. For the interaction I whose pseudopotentials are shown by cross symbols, we set the priority parameters as $\kappa_{0}=-1$ and $\kappa_{J}=0$ for $J \neq 0$. Namely we minimized the difference, $\left|V_{0}-V_{0}^{\mathrm{C}}\right|$, between the longest-range components to mimic the long-range nature of the Coulomb interaction. As the inequalities imposed in the linear programming

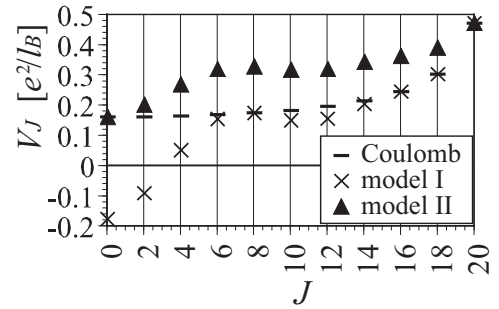

Fig. 1. Pseudopotentials for negative-sign-free interactions and the Coulomb interaction within the lowest Landau level with $N_{s}=2 s+1=22$. Only physical components for odd $2 s-J$ are shown. The pseudopotentials of negative-sign-free model interaction named I are obtained by the linear programming method and are shown by cross symbols. The pseudopotentials of interaction II shown by closed triangles are obtained by applying uniform shifts and rescaling to those of interaction I.

method, we used the following ones: $V_{2 s-1} \geq V_{2 s-1}^{\mathrm{C}}$ (the shortest-range component) and $V_{J} \leq V_{J}^{\mathrm{C}}$ for $J \neq 2 s-1$. This is because the Laughlin state favors prominently large short-range components. Pseudopotentials of model interaction I agree well with those of the Coulomb interaction on short-range components, but do not on longrange ones. In fact, the long-range components shift downward from those of the Coulomb interaction and eventually become even negative.

In order to make improvements on the interaction I, we perform the following operations on its pseudopotentials: (i) uniform shift of pseudopotentials, $V_{J} \rightarrow V_{J}+\left|V_{0}^{\mathrm{I}}\right|$, (ii) energy rescale, $V_{J} \rightarrow V_{J} \times\left(V_{2 s-1}^{\mathrm{C}}-V_{0}^{\mathrm{C}}\right) /\left(V_{2 s-1}^{\mathrm{I}}-V_{0}^{\mathrm{I}}\right)$, and (iii) another uniform shift of pseudopotentials, $V_{J} \rightarrow$ $V_{J}+V_{0}^{\mathrm{C}}$. As a result of these sequential operations, we obtain another interaction named II, whose pseudopotentials are given by $V_{J}^{\mathrm{II}}=V_{0}^{\mathrm{C}}+\left(V_{J}^{\mathrm{I}}-V_{0}^{\mathrm{I}}\right) \times$ $\left(V_{2 s-1}^{\mathrm{C}}-V_{0}^{\mathrm{C}}\right) /\left(V_{2 s-1}^{\mathrm{I}}-V_{0}^{\mathrm{I}}\right)$. We note here that uniform shifts of pseudopotentials make no influence on the electronic states, because the change, $V_{J} \rightarrow V_{J}+v$ for all $J$, involves just a constant term in the Hamiltonian as $\frac{1}{2} v \sum_{J M} A_{J M}^{\dagger} A_{J M}=\frac{1}{2} v N_{e}\left(N_{e}-1\right)$. Furthermore these operations do not break the negative-sign-free character of $\chi_{K}$, because uniform shifts of $V_{J}$ bring about only the change in $\chi_{K=0}$ and energy rescale keeps the sign of $\chi_{K}$. Thus the interaction II is also free of negative-sign.

The pseudopotentials of interaction II are shown by closed triangles in Fig.1. The energy scales of interaction II and Coulomb interaction are the same, because $V_{2 s-1}-V_{0}$ gives the energy scale. The character of interaction II is similar to that of the Coulomb interaction on short- and long-range components, while the difference between pseudopotentials of the interaction II and Coulomb interaction is not so small on middle-range ones. In spite of this disagreement on middle-range ones, it is shown later that the interaction II leads to the ground-state properties qualitatively similar to those of the Coulomb-interacting system.

Figure 2 shows the results of zero-temperature QMC calculations for the QH system with $2 s=21$ and $N_{e}=8$ $(\nu=1 / 3)$. These calculations were done by using the negative-sign-free interaction II in Fig.1. Calculated results for three imaginary-time slice widths, $\Delta \beta=1 / 4$, 

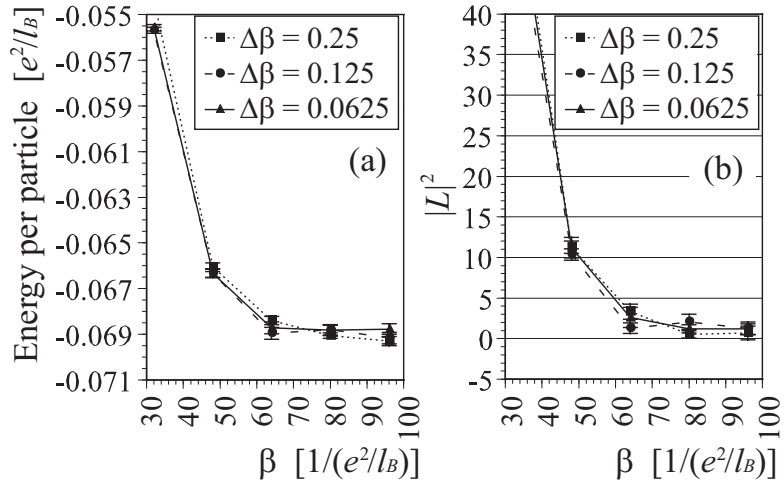

Fig. 2. Expectation values of (a) energy per particle and (b) total angular momentum by the zero-temperature QMC calculations of the QH system with $2 s=21$ and $N_{e}=8(\nu=1 / 3)$. The unit of energy is $e^{2} / l_{B}\left(l_{B}\right.$ : the magnetic length), $\beta$ the inverse temperature, and $\Delta \beta$ is the width of imaginary-time slice. The energy contribution by the positive-charge background is also taken into account.

$1 / 8$, and $1 / 16\left[1 /\left(e^{2} / l_{B}\right)\right]$ are almost the same. Hence the numerical errors introduced by the Suzuki-Trotter approximation are not so important for $\Delta \beta \lesssim 1 / 4$. For the inverse temperature $\beta \gtrsim 60 /\left(e^{2} / l_{B}\right)$, both expectation values of energy and angular momentum are almost saturated. The energy gap for the interaction II is found to be $\Delta E \simeq 0.09\left(e^{2} / l_{B}\right)$ by the exact diagonalization method. The inverse temperature needed for convergence is so high $(\beta \gtrsim 6 / \Delta E)$. This seems to be due to a high density of states for $E \gtrsim \Delta E$.

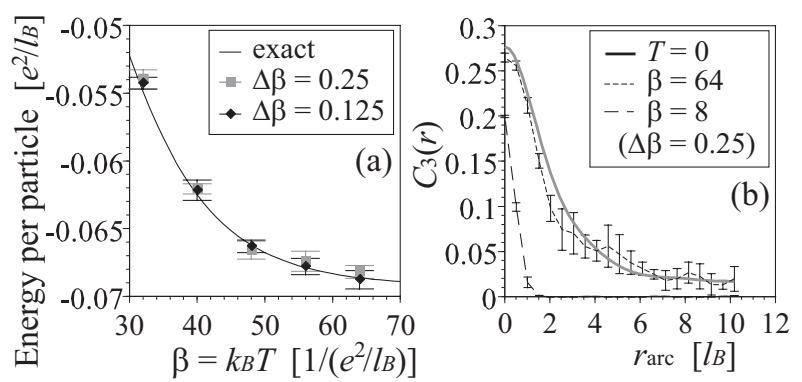

Fig. 3. Expectation values of (a) energy per particle and (b) ODLRO correlation function $C_{3}(r)$. Results by the exact diagonalization and finite-temperature QMC calculations are shown for the QH system with $2 s=21$ and $N_{e}=8(\nu=1 / 3) \cdot r_{\text {arc }}$ denotes the arc-distance between $\mathbf{r}$ and the north-pole on the Haldane spherical surface.

Figure 3(a) shows the $\beta$-dependence of energy expectation value per particle in the canonical ensemble. The results by the exact diagonalization and QMC calculations are in good agreement. For $\beta \gtrsim 60 /\left(e^{2} / l_{B}\right)$, the expectation values of energy per particle are almost saturated as well as in Fig.2(a). Since the existence of the offdiagonal long-range order (ODLRO) is one of the most remarkable properties of the Laughlin state, ${ }^{17}$ we calculated the ODLRO correlation function in the $\nu=1 / 3$ $\mathrm{QH}$ system with interaction II. The correlation function is defined ${ }^{18}$ by $C_{3}\left(\mathbf{r}^{\prime}-\mathbf{r}\right)=\left\langle A^{\dagger}\left(\mathbf{r}^{\prime}\right) A(\mathbf{r})\right\rangle$, where $A(\mathbf{r})$ is the annihilation operator of a composite boson (an electron with three flux quanta attached to it). Figure 3(b) shows $C_{3}(\mathbf{r})$ for $\beta=8$ and $64\left[1 /\left(e^{2} / l_{B}\right)\right]$ obtained by QMC calculations as well as that at $T=0$ obtained by the exact diagonalization. At zero temperature, $C_{3}(\mathbf{r})$ remains finite for large separation $r$. This result suggests that the ground state for the interaction II should have the ODLRO. For finite temperatures $\beta \lesssim 8$, the ODLRO correlation rapidly vanishes with increasing separation $r$, while it survives at $\beta=64 \simeq 6 / \Delta E$ as in case of $T=0$. This is because the contributions of excited states to $C_{3}(r)$ is negligible for temperatures quite lower than $\Delta E$.

We comment on the validity of negative-sign-free interaction II as a substitute for the Coulomb interaction. By the exact diagonalization method, we confirmed that the overlap between the two ground states for the interaction II and Coulomb interaction is not large $(\sim 0.35)$ for $\nu=1 / 3$. However, the existence of the ODLRO in the ground state and that of energy gap above it suggest that an imperfect Bose condensation of composite bosons exists for interaction II, although it is not so perfect as that in the Laughlin state. Thus we believe that the ground state for interaction II contains the essential properties of the Laughlin state.

The authors thank Y. Kato, A.H. MacDonald, and A. Muramatsu for useful discussion. S.S. acknowledges support by Research Fellowship for young scientists of JSPS. The present work is supported by Grant-in-Aid for Scientific Research (Grant No.1406899 and No.14740181) by the Ministry of Education, Culture, Sports, Science and Technology of Japan.

1) D.C. Tsui, H.L. Stormer, and A.C. Gossard, Phys. Rev. Lett. 48 (1982) 1559.

2) W. Pan, J.-S. Xia, V.Shvarts, D.E. Adams, H.L. Stormer, D.C. Tsui, L.N. Pfeiffer, K.W. Baldwin, and K.W. West, Phys. Rev. Lett. 83 (1999) 3530.

3) The Quantum Hall Effect second edition, edited by R.E. Prange and S.M. Girvin (Springer, 1990).

4) R.B. Laughlin, Phys. Rev. Lett. 50 (1983) 1395.

5) J.K. Jain, Phys. Rev. Lett. 63 (1989) 199.

6) G. Moore and N. Read, Nucl. Phys. B360 (1991) 362.

7) B.I. Halperin, P.A. Lee and N. Read, Phys. Rev. B47 (1993) 7312 .

8) E.H. Rezayi and N. Read, Phys. Rev. Lett. 73 (1994) 900.

9) A.A. Koulakov, M.M. Fogler, and B.I. Shklovskii, Phys. Rev. Lett. 76 (1996) 499.

10) D. Yoshioka, B.I. Halperin, and P.A. Lee, Phys. Rev. Lett. 50 (1983) 1395.

11) N. Shibata and D. Yoshioka, Phys. Rev. Lett. 86 (2001) 5755.

12) F.D.M. Haldane, Phys. Rev. Lett. 51 (1983) 605.

13) G. Fano, F. Ortolani, and E. Colombo, Phys. Rev. B34 (1986) 2670.

14) E.Y. Loh Jr. and J.E. Gubernates, in Electronic Phase Transitions, edited by W. Hanke and Yu. V. Kopaev (Elsevier Science Publishers B. V., New York, 1992).

15) S.E. Koonin, D.J. Dean, K. Langanke, Phys. Rep. 278 (1997) 1.

16) G.H. Lang, C.W. Johnson, S.E. Koonin, and W.E. Ormand, Phys. Rev. C 48 (1993) 1518.

17) S.M. Girvin and A.H. MacDonald, Phys. Rev. Lett. 58 (1987) 1252.

18) E.H. Rezayi and F.D.M. Haldane, Phys. Rev. Lett. 61 (1988) 1985. 